\title{
Paratesticular rhabdomyosarcoma of a young adult
}

\author{
Gorana Matovina-Brko, Borislava Nikolin, Lazar Popovic, Zoran Nikin, Svetlana Popovic-Petrovic, Darjana Jovanovic
}

Oncology Institute of Vojvodina, Sremska Kamenica, Serbia

Medical Faculty, University of Novi Sad, Novi Sad, Serbia

Contemp Oncol (Pozn) 2015; 19 (3): 252-256 DOI: $10.5114 /$ wo.2015.52661

Sarcomas represent large and diverse group of malignant tumours, originating from mesenchymal cells. Nearly $15 \%$ of all childhood tumours and about $1 \%$ of all tumours in adults are sarcomas [1]. The incidence of adult soft tissue sarcomas in Europe is 5/100,000 [2]. Rhabdomyosarcoma accounts for $4.6 \%$ of all soft tissue sarcomas and it is the most common paediatric sarcoma, while it is extremely rare in adults [3].

The majority of scrotal neoplasms originate from the testicle itself. However, some may arise from mesenchymal elements of paratesticular tissue: spermatic cord, epididymis, and testicular envelopes. Only about $30 \%$ of all paratesticular neoplasms are malignant. Their real origin in comparison to anatomical structures is somewhat hard to determine; however, it is said that spermatic cord is the most common site (90\%) [4]. The most common paratesticular neoplasm is soft tissue sarcoma: leiomyosarcoma, rhabdomyosarcoma, and liposarcoma being at the top of the list [5].

We report a rare case of a 25 -year-old male who was admitted at our institution after surgical treatment in a small regional centre. Our diagnosis based on delivered paraffin-embedded tumour tissue samples was: Paratesticular pleomorphic rhabdomyosarcoma, high grade. Pathohistological report was: Tumour tissue consists of fusiform, oval, polygonal, spindle and numerous bizarre large rhabdomyoblasts, including multinucleated ones. Nuclei are hyperchromatic. In bizarre rhabdomyoblasts cytoplasm is abundant, eosinophilic, granular, rich in thick and thin filaments (Fig. 1). Tumour cells are forming nests and sheets. Stroma is variably myxoid with minor sclerotic areas. Microcystic formations are focally present. Minor elements of embryonal (spindle cell) and alveolar rhabdomyosarcoma as well as sclerosing rhabdomyosarcoma are also present (Fig. 2). Tumour is well vascularised. Mitotic activity is high (over 10 mitosis per 10 high power fields). Immunophenotype: vimentin + (monoclonal antibody, clone V9, DAKO, visualisation kit FLEX), desmin + (monoclonal antibody, clone D33, Lab Vision IHC System Solution, visualisation kit FLEX) (Figs. 3 and 4) and actin + (monoclonal antibody, clone HHF35, DAKO, visualisation kit FLEX), MyoD1 - (monoclonal antibody, clone 5.8A, DAKO, visualisation kit LP detection system), and S-100 protein - (polyclonal antibody, DAKO, visualisation kit FLEX, CD34-, CD99-, CD117-). The patient was initially treated in a small regional centre and was referred to a medical oncologist only after the first relapses, three months after the surgery. He was in a good physical shape, with no complications. He did not have any significant personal or family medical history. Serum alphaphetoprotein, lactate dehydrogenases, and



Fig. 1. Pleomorphic rhabdomyosarcoma. Staining by hematoxylineosin stain (magnification 200x): Bizarre rhabdomyoblasts with abundant, eosinophilic, granular cytoplasm, rich in thick and thin filaments

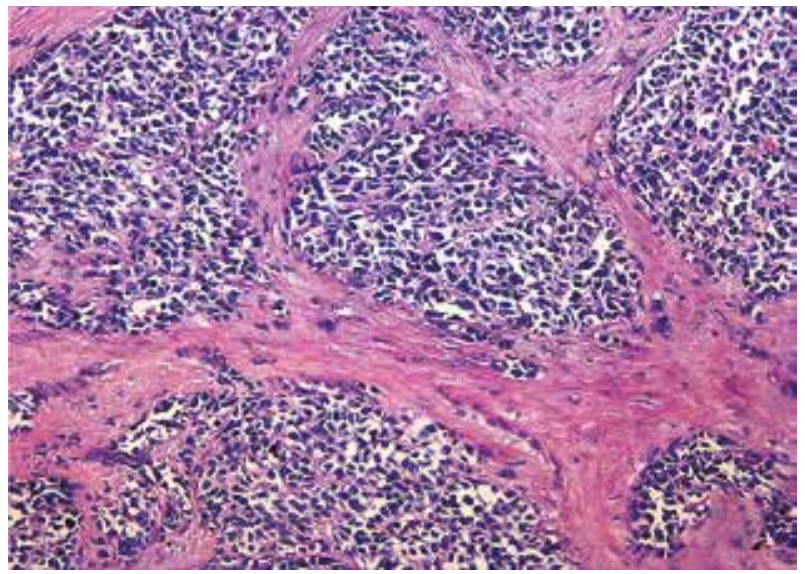

Fig. 2. Sclerosing rhabdomyosarcoma. Staining by hematoxylin-eosin stain (magnification 100x): Nests and sheets of tumor cells, sclerotic stroma 




Fig. 3. Desmin immunohistochemistry (magnification 200x). Cytoplasmic positivity of tumor cells for desmin

$\beta$-human chorionic gonadotropin were normal. Chest and abdominal CTs were normal. A CT of the pelvis described relapse in the right inguinal canal. He underwent surgery at the Urology Clinic: bilateral retroperitoneal and right inguinal lymphadenectomy and right orchiectomy. He was administered adjuvant treatment with doxorubicin, four cycles, and four more cycles of doxorubicin and ifosphamide due to retroperitoneal lymphadenomegaly. Post-chemotherapy reassessment showed only the persistent retroperitoneal infiltrates. He was reoperated, and histopathology confirmed the previous diagnosis. Postoperative imaging showed progression of the disease in lungs, liver, retroperitoneum, and bone structures. The patient was in good physical condition, Karnofsky status 100\%. He was planned and prepared to be included in a running clinical trial on soft tissue sarcoma. However, he failed screening for inclusion in the trial due to high liver enzymes and impaired kidney function, and was reassigned to the best supportive care. He died a few weeks later, 22 months after the initial diagnosis.

Paratesticular rhabdomyosarcoma is an extremely rare malignancy of adulthood and constitutes $7 \%$ of all rhabdomyosarcoma and $6 \%$ of all non-germinal intrascrotal neoplasms [5].

Two incidence peaks of paratesticular rhabdomyosarcoma occur, the first between age two and six, and the second between fifteen and nineteen years of age. $70 \%$ of all cases occur before the age of 10 [6]. Therefore, most of the data on paratesticular rhabdomyosarcoma can be found in paediatric sources only. Available data on this extremely infrequent localisation of an even more rare tumour of adulthood is insufficient and scarce, mostly presented as case reports or single institution experiences with small numbers of patients, different treatment modalities, and with different stages of the disease [7-13].

Paratesticular tumours are commonly presented as palpable masses. Concerning its surface presentation, in comparison with sarcomas situated deeper in tissues or retroperitoneum, they are frequently diagnosed earlier. This should contribute to their better prognosis in comparison to the other sites. The patient first came to the doctor



Fig. 4. Desmin immunohistochemistry (magnification 200x). Cytoplasmic positivity of tumor cells for desmin

two months after the initial symptoms. Time to diagnosis is longer in older children and adolescents than in younger children, which can explain the incidence of nodal involvement and distant metastases [14].

The most common histopathology of paratesticular rhabdomyosarcoma is spindle cell subtype of embryonal rhabdomyosarcoma presented in a younger population of patients with its typical paratesticular localisation. According to single institution experiences and the Intergroup Rhabdomyosarcoma Study (IRS) I and II, up to 95\% of the paratesticular rhabdomyosarcoma are of embryonic type $[15,16]$.

Our patient had a predominantly pleomorphic type of rhabdomyosarcoma, but it also contained elements of embryonal (spindle cell variant) as well as alveolar rhabdomyosarcoma. Pleomorphic type is the rarest type, typical for adults older than 45 years. It is most commonly localised in striated muscles of extremities and has the worst prognosis [17]. A PubMed search found a few cases of older patients with pleomorphic paratesticular rhabdomyosarcoma, while most adult as well as paediatric patients had embryonal type $[8,13]$. The sample material also contained elements

Table 1. IRSG Postsurgical Grouping Classification

\begin{tabular}{|c|c|}
\hline \multicolumn{2}{|c|}{ IRSG Postsurgical Grouping Classification } \\
\hline Group 1 & $\begin{array}{l}\text { Localized disease, completely excised, no microscopic } \\
\text { residual }\end{array}$ \\
\hline A & Confined to site of origin, completely resected \\
\hline B & Infiltrating beyond site of origin, completely resected \\
\hline Group 2 & Total gross resection \\
\hline A & $\begin{array}{l}\text { Gross resection with evidence of microscopic local } \\
\text { residual }\end{array}$ \\
\hline B & $\begin{array}{l}\text { Regional disease with involved lymph nodes, } \\
\text { completely resected with no microscopic residual }\end{array}$ \\
\hline C & Microscopic local and/or nodal residual \\
\hline Group 3 & Incomplete resection or biopsy with gross residual \\
\hline Group 4 & Distant metastases \\
\hline
\end{tabular}


Table 2. IRSG Presurgical Staging Classification

\begin{tabular}{|c|c|c|c|c|c|}
\hline \multicolumn{6}{|c|}{ IRSG Presurgical Staging Classification } \\
\hline Stage & Sites & Tumor $(\mathrm{T})$ & Size & Node $(\mathrm{N})$ & Metastases (M) \\
\hline 1 & $\begin{array}{l}\text { Orbit, head and neck (excluding parameningeal), genitourinary: } \\
\text { nonbladder/nonprostate }\end{array}$ & $\mathrm{T}_{1}$ or $\mathrm{T}_{2}$ & $\begin{array}{l}a \text { or } \\
b\end{array}$ & $\begin{array}{l}\mathrm{N}_{0}, \mathrm{~N}_{1} \text {, or } \\
\mathrm{Nx}\end{array}$ & $M_{0}$ \\
\hline$\|$ & $\begin{array}{l}\text { Bladder/prostate, extremity, cranial, parameningeal, other (includes } \\
\text { trunk, retroperitoneum, and so on) }\end{array}$ & $\mathrm{T}_{1}$ or $\mathrm{T}_{2}$ & a & $\mathrm{N}_{0}$ or $\mathrm{N}_{\mathrm{x}}$ & $M_{0}$ \\
\hline III & $\begin{array}{l}\text { Bladder/prostate, extremity, cranial parameningeal, other (includes } \\
\text { trunk, retroperitoneum, and so on) }\end{array}$ & $\mathrm{T}_{1}$ or $\mathrm{T}_{2}$ & $a b$ & $\begin{array}{l}\mathrm{N}_{1} \mathrm{~N}_{0}, \mathrm{~N}_{1} \\
\quad \text { or } \mathrm{Nx}\end{array}$ & $M_{0}$ \\
\hline IV & All & $\mathrm{T}_{1}$ or $\mathrm{T}_{2}$ & $\begin{array}{l}a \text { or } \\
b\end{array}$ & $\mathrm{~N}_{0}$ or $\mathrm{N}_{1}$ & $M_{1}$ \\
\hline
\end{tabular}

Tumor: $T_{1}$, confined to anatomic site of origin, (a) $\leq 5 \mathrm{~cm}$ in diameter in size, (b) $>5 \mathrm{~cm}$ in diameter in size; $T_{2}$, extension and/or fixative to surrounding tissue, (a) $\leq 5 \mathrm{~cm}$ in diameter in size, (b) $>5 \mathrm{~cm}$ in diameter in size; regional nodes: $N_{0}$, regional nodes not clinically involved; $N_{1}$, regional nodes clinically involved by neoplasm; $N_{x^{\prime}}$ clinical status of regional nodes unknown; metastasis: $M_{0}$, no distant metastasis; $M_{1}$, metastasis present.

of sclerosing rhabdomyosarcoma, a relatively new entity, still not recognised enough, first described in the year 2000 [18]. A few small studies and individual case reports imply that sclerosing rhabdomyosarcoma is probably correlated to embryonic rhabdomyosarcoma [19]. A PubMed search did not find any reported cases of paratesticular rhabdomyosarcoma with this new histopathological entity. Tumour tissue was MyoD1 negative in both primary and metastatic samples. MyoD1 (myogenic determination factor) shows excellent although not 100\% specificity (about 90\%) and sensitivity (about 97\%) for skeletal muscles and rhabdomyosarcomas [20, 21] providing adequate laboratory preparation and formalin fixation [22]. However, the obtained tumour material did not have adequate formalin fixation (but alcohol fixation) since it was processed in another hospital with no immunohistochemistry. Primary differential diagnosis could be leiomyosarcoma or malignant fibrous histiocytoma; however, the morphologic characteristics of the tumour speak in favour of rhabdomyosarcoma. A less likely tumour could be pleomorphic liposarcoma or osteosarcoma, but these are discarded due to positive muscle markers. Malignant triton tumour (a type of malignant schwannoma) can have muscle differentiation as well, but it does not present this kind of morphologic characteristics [5].

The IRS group (IRSG) classifies rhabdomyosarcoma into four clinical groups and four stages of disease that guide the treatment and are related to prognosis (Table 1 and 2). According to IRGS, four study experience favourable prognostic factors are: undetectable distant metastases at diagnosis, primary sites in the orbit and non-parameningeal head/neck, genitourinary non-bladder/prostate regions, grossly removal of localised tumour at the time of diagnosis, embryonal/botryoid histology, and tumour size $\leq 5 \mathrm{~cm}$ and age younger than 10 years at diagnosis [23].

Paratesticular rhabdomyosarcoma patients in IRS groups I-III have a five-year survival rate of $94.6 \%$, while IRS group IV has an extremely poor prognosis with up to $22.2 \%$ five-year survival [14]. The most common sites of metastases forming paratesticular rhabdomyosarcomas are regional lymph nodes, lungs, cortical bone, and bone marrow [24]. Retroperitoneal metastases occur in up to $40 \%$ of the patients [14].
In our patient, at the time of diagnosis there was no visible spread of the disease (stage I, group I) according to the IRS classification system. However, the patient was assigned only for follow up. Three months after the surgery, inguinal relapse occurred. At that time, CT confirmed enlarged retroperitoneal as well as inguinal lymph nodes, so right orchiectomy and right inguinal and bilateral retroperitoneal lymph node dissection (RPLND) were performed. The patient was classified as IRS IIB. According to The German and Italian cooperative group experience with 216 paediatric patients with paratesticular rhabdomyosarcoma treated over 20 years, completely resectable cases in children had excellent outcome, even in those whose first surgical procedure was not satisfactory. This group considered that inguinal incision mandatory and re-excision should be recommended in cases of initial trans-scrotal approach [14].

In cases of paratesticular rhabdomyosarcoma in childhood, unilateral nerve sparing dissection should be performed in all children older than 10 years, as well in those with clinically positive lymph nodes [25]. Use of only CT scan of RPLN led to a decrease in identification of RPLN involvement in IRS-IV and a higher rate of regional relapse as compared with IRS-III. Wiener et al. therefore suggest ipsilateral RPLND as part of routine staging of adolescents with paratesticular rhabdomyosarcoma, and those with positive lymph nodes require intensified chemotherapy as well as nodal irradiation [26]. Considering the aggressive biology of the disease and the necessity of adequate staging, retroperitoneal lymph node sampling of the ipsilateral chain should be obligatory in all adult patients with paratesticular rhabdomyosarcoma [27]. Positive nodes higher than the renal vessels are considered as disseminated metastatic disease. Inguinal nodes are rarely involved and are biopsied only if clinically positive or if the scrotum is invaded by tumour. Inguinal nodes are not considered regional [28].

Adjuvant chemotherapy should be considered in all patients with paratesticular rhabdomyosarcoma. The majority of reported protocols are doxorubicin and ifosphamide based, but also consist of etoposide, vincristine, actinomycin-D, cyclophosphamide, and cisplatin. According to the 
Minimal Clinical Recommendations of ESMO and NCCN, first-line chemo for soft tissue sarcoma is doxorubicin, and considering overall survival there are no convincing data that polychemotherapy is more effective in comparison with doxorubicin alone. However, a combination of doxorubicin with ifosphamide gives a better response rate (RR) [1, 2], being up to $86 \%$ according to the results of recent studies of extremity-localised rhabdomyosarcoma in adult patients [29].

Even after surgery and adjuvant chemotherapy our patient developed regional lymph node and distant visceral and skeletal metastases. He died shortly after diagnosis of metastatic disease, the pace of which was furious, with no opportunity of enrolment in a planned clinical trial. In spite of applied chemotherapy, for adult patients with rhabdomyosarcoma of extremities, overall survival ranges from $25-45 \%[30,31]$.

Local radiotherapy is recommended in addition to systemic treatment in all patients with histopathologically positive lymph nodes and microscopic residual disease [15]. In their study of paratesticular rhabdomyosarcoma a German and Italian cooperative group administered external beam irradiation in concomitance with chemotherapy, doses ranging from 32 Gy to 65 Gy - standard fractionation was used as well as hyperfractionation. However, according to their experience, no significant difference was noted among N1 patients who were and were not treated with radiotherapy [14].

In conclusion, although extremely rare in the adult population in comparison with paediatric population, rhabdomyosarcoma in general represents a more aggressive and more malignant neoplasm [32]. Considering the more aggressive histopathological variety in adults, as well as the fact that rhabdomyosarcoma in childhood, adolescence, and adulthood is presented with distinctive biology, adult rhabdomyosarcoma represents a unique entity. What we can surely state is that paratesticular rhabdomyosarcoma has to be treated in highly specialised oncological institutions, and the therapeutic approach has to be multimodal and has to involve an experienced surgeon, medical oncologist, radiotherapist, pathologist, and radiologist. As with soft tissue sarcoma in general, a final solution lies in the future, we hope. Some new treatment modalities for sarcomas are developing and are the subject of clinical trials. However, so far none of them have brought any significant step forward in effective treatment of these malignancies (mTOR inhibitors, tyrosine kinase inhibitors, heat shock protein inhibitors).

The authors declare no conflict of interest.

\section{References}

1. NCCN. Clinical practice guidelines in oncology: soft tissue sarco ma v.2.2008. Available at: http://www.nccn.org/professionals/pysician_gls/PDF/sarcoma.pdf.

2. Casali PG, Blay JY; ESMO/CONTICANET/EUROBONET Consensus Panel of experts. Soft tissue sarcomas: ESMO Clinical Practice
Guidelines for diagnosis, treatment and follow-up. Ann Oncol 2010; 21 Suppl 5: 198-203.

3. Toro JR, Travis LB, Wu HJ, Zhu K, Fletcher CD, Devesa SS. Incidence patterns of soft tissue sarcomas, regardless of primary site, in the surveillance, epidemiology and end results program, 1978-2001: An analysis of 26,758 cases. Int J Cancer 2006; 119: 2922-30.

4. Richie JP. Neoplasm of testis. In: Campbell's Urology. Walsh PC, Retik AB, Vaughan ED, Wein AJ (eds.). $7^{\text {th }}$ ed. Vol. III. WB Saunders, Philadelphia 1998; 2411-52.

5. Khoubehi B, Mishra V, Ali M, Motiwala H, Karim O. Adult paratesticular tumours. BJU Int 2002; 90: 707-15.

6. Weiss SW and Goldblum JR Rhabdomyosarcoma. In Enzinger and Weiss's Soft Tissue Tumors, Weiss SW, Goldblum JR (eds.). CV Mosby, St. Louis 2001; 785-835.

7. Silvestris N, Zito FA, Resta L, Popescu O, Rossi R, Montemurro S, Colucci G. Unique case of giant adult paratesticular spindle cell rhabdomyosarcoma. Urology 2009; 73: 500-2

8. Kourda N, El Atat R, Derouiche A, Bettaib I, Baltagi S, Zermani R. Paratesticular pleomorphic rhabdomyosarcoma in an adult: diagnosis and management. Cancer Radiother 2007; 11: 280-3.

9. Kizer WS, Dykes TE, Brent EL, Chatham JR, Schwartz BF. Paratesticular spindle cell rhabdomyosarcoma in an adult. J Urol 2001; 166: 606-7.

10. Kosan M, Gonulalan U, Ugurlu O, Seckin S, Adsan O. Embryonal paratesticular rhabdomyosarcoma: a case of young adult patient who has inguinal relapse. Int Urol Nephrol 2007; 39: 1159-61.

11. Albino G, Nenna R, Inchingolo CD, Marucco EC. Hydrocele with surprise. Case report and rewiew of literature. Arch Ital Urol Androl 2010; 82: 287-90.

12. Haga K, Kashiwagi A, Nagamori S, Yamashiro K. Adult paratesticular rhabdomyosarcoma. Nat Clin Pract Urol 2005; 2: 398-402.

13. Demir A, Onol FF, Türkeri L. Paratesticular pleomorphic rhabdomyosarcoma in an adult. Int Urol Nephrol 2004; 36: 577-8.

14. Ferrari A, Bisogno G, Casanova M, et al. Paratesticular rhabdomyosarcoma: report from the Italian and German Cooperative Group. J Clin Oncol 2002; 20: 449-55.

15. Ferrari A, Casanova M, Massimino M, Luksch R, Piva L, Fossati-Bellani F. The management of paratesticular rhabdomyosarcoma: a single institutional experience with 44 consecutive children. J Urol 1998; 159: 1031-4.

16. Raney RB Jr, Tefft M, Lawrence W Jr, Ragab AH, Soule EH, Beltangady M, Gehan EA. Paratesticular sarcoma in childhood and adolescence. A report from the Intergroup Rhabdomyosarcoma Studies I and II, 1973-1983. Cancer 1987; 60: 2337-43.

17. Newton WA Jr, Soule EH, Hamoudi AB, et al. Histopathology of childhood sarcomas, Intergroup Rhabdomyosarcoma Studies I and II: clinicopathologic correlation. J Clin Oncol 1988; 6: 67-75.

18. Mentzel T, Katenkamp D. Sclerosing, pseudovascular rhabdomyosarcoma in adults: clinicopathological and immunohistochemical analysis of three cases. Virchows Arch 2000; 436: 305-11.

19. Wang J, Tu X, Sheng W. Sclerosing rhabdomyosarcoma: a clinicopathologic and immunohistochemical study of five cases. Am J Clin Pathol 2008; 129: 410-5.

20. Morrotti RA, Nicol KK, Parham DM, Teot LA, Moore J, Hayes J, Meyer W, Oualman S); Children's Oncology Group. An immunohistochemical algorithm to facilitate diagnosis and subtyping of rhabdomyosarcoma: the Children's Oncology Group experience. Am J Surg Pathol 2006; 30: 962-8

21. Cessna MH, Zhou H, Perkins SL, Tripp SR, Layfield L, Daines C, Coffin CM. Are myogenin and myoD1 expression specific for rhabdomyosarcoma? A study of 150 cases, with emphasis on spindle cell mimics. Am J Surg Pathol 2001; 25: 1150-7.

22. Parham DM, Ellison DA. Rhabdomyosarcomas in adults and children: an update. Arch Pathol Lab Med 2006; 130: 1454-65.

23. Raney RB, Anderson JR, Barr FG, et al. Rhabdomyosarcoma and undifferentiated sarcoma in the first two decades of life: a selective review of intergroup rhabdomyosarcoma study group experience and rationale for Intergroup Rhabdomyosarcoma Study V. J Pediatr Hematol Oncol 2001; 23: 215-20.

24. Akbar SA, Syyet TA, Jafri SZ, Hasteh F, Neill JS. Multimodality imaging of paratesticular neoplasms and their rare mimics. Radiographics 2003; 23: 1461-76 
25. Breneman JC, Wiener ES. Issues in the local control of rhabdomyo sarcoma. Med Pediatr Oncol 2000; 35: 104-9.

26. Wiener ES, Anderson JR, Ojimba JI, et al. Controversies in the management of paratesticular rhabdomyosarcoma: is staging retroperitoneal lymph node dissection necessary for adolescents with resected paratesticular rhabdomyosarcoma? Semin Pediatr Surg 2001; 10: 146-52.

27. Hermans BP, Foster RS, Bihrle R, Little S, Sandler A, Einhorn LH, Donohue JP. Is retroperitoneal lymph node dissection necessary for adult paratesticular rhabdomyosarcoma? J Urol 1998; 160: 2074-7.

28. Rodeberg DA, Paidas CN, Lobe TL, Brown K, Andrassy RJ, Crist WM, Wiener ES. Surgical principles for children/adolescents with newly diagnosed rhabdomyosarcoma: a report from the Soft Tissue Sarcoma Committee of the Children's Oncology Group. Sarcoma 2002; 6: 111-22.

29. Ogilvie CM, Crawford EA, Slotcavage RL, King J, Lackman RD, Hartner L, Staddon AP. Treatment of adult rhabdomyosarcoma. Am J Clin Oncol 2010; 33: 128-31.

30. Esnaola NF, Rubin BP, Baldini EH, Vasudevan N, Demetri GD, Fletch er CD, Singer S. Response to chemotherapy and predictors of survival in adult rhabdomyosarcoma. Ann Surg 2001; 234: 215-23.

31. Ferrari A, Dileo P, Casanova M, et al. Rhabdomyosarcoma in adults: a retrospective analysis of 171 patients treated at a single institution. Cancer 2003; 98: 571-80.

32. Hawkins WG, Hoos A, Antonescu CR, et al. Clinicopathologic analysis of patients with adult rhabdomyosarcoma. Cancer 2001; 91: 794-803.

\section{Address for correspondence}

Gorana Matovina-Brko, MD

Oncology Institute of Vojvodina,

Department of Medical Oncology,

Put doktora Goldmana 4

21204 Sremska Kamenica, Serbia

e-mail: gorana021@yahoo.com

tel. +38162404818

$\mathrm{fax}+381216613741$

Submitted: 27.05 .2013

Accepted: $\quad 5.08 .2013$ 